\title{
Cryo-EM structures of tau filaments from Alzheimer's disease with PET ligand APN-1607
}

\author{
Yang Shi ${ }^{1}$ - Alexey G. Murzin ${ }^{1}$ - Benjamin Falcon ${ }^{1} \cdot$ Alexander Epstein $^{1} \cdot$ Jonathan Machin ${ }^{1} \cdot$ Paul Tempest $^{2}$. \\ Kathy L. Newell ${ }^{3}$. Ruben Vidal ${ }^{3} \cdot$ Holly J. Garringer $^{3} \cdot$ Naruhiko Sahara $^{4} \cdot$ Makoto Higuchi $^{4} \cdot$ Bernardino Ghetti $^{3}$. \\ Ming-Kuei Jang ${ }^{2} \cdot$ Sjors H. W. Scheres ${ }^{1} \cdot$ Michel Goedert $^{1}$
}

Received: 14 December 2020 / Revised: 4 March 2021 / Accepted: 4 March 2021 / Published online: 16 March 2021

(c) The Author(s) 2021, corrected publication 2021

\begin{abstract}
Tau and $\mathrm{A} \beta$ assemblies of Alzheimer's disease (AD) can be visualized in living subjects using positron emission tomography (PET). Tau assemblies comprise paired helical and straight filaments (PHFs and SFs). APN-1607 (PM-PBB3) is a recently described PET ligand for AD and other tau proteinopathies. Since it is not known where in the tau folds PET ligands bind, we used electron cryo-microscopy (cryo-EM) to determine the binding sites of APN-1607 in the Alzheimer fold. We identified two major sites in the $\beta$-helix of PHFs and SFs and a third major site in the C-shaped cavity of SFs. In addition, we report that tau filaments from posterior cortical atrophy (PCA) and primary age-related tauopathy (PART) are identical to those from AD. In support, fluorescence labelling showed binding of APN-1607 to intraneuronal inclusions in AD, PART and PCA. Knowledge of the binding modes of APN-1607 to tau filaments may lead to the development of new ligands with increased specificity and binding activity. We show that cryo-EM can be used to identify the binding sites of small molecules in amyloid filaments.
\end{abstract}

Keywords Positron emission tomography $\cdot$ Alzheimer's disease $\cdot$ Posterior cortical atrophy $\cdot$ Primary age-related tauopathy $\cdot$ Immunopurification $\cdot$ Electron cryo-microscopy

\section{Introduction}

Assembly of a small number of soluble proteins into insoluble amyloid filaments underlies the majority of age-related neurodegenerative diseases [20]. Tau is the most commonly affected of these proteins. It assembles into filaments in a

Sjors H. W. Scheres and Michel Goedert jointly supervised this work.

Sjors H. W. Scheres

scheres@mrc-lmb.cam.ac.uk

$\triangle$ Michel Goedert

mg@mrc-lmb.cam.ac.uk

MRC Laboratory of Molecular Biology, Cambridge, UK

2 APRINOIA Therapeutics, Taipei, Taiwan

3 Department of Pathology and Laboratory Medicine, Indiana University School of Medicine, Indianapolis, IN 46202, USA

4 National Institute of Radiological Sciences, National Institutes for Quantum and Radiological Science and Technology, Chiba 263-8555, Japan number of diseases, including Alzheimer's disease (AD), chronic traumatic encephalopathy (CTE), progressive supranuclear palsy (PSP), globular glial tauopathy (GGT), corticobasal degeneration (CBD) and Pick's disease (PiD). Six tau isoforms are expressed in the adult human brain. Three isoforms have three microtubule-binding repeats, whereas the other three have four repeats each. All six tau isoforms assemble in AD and CTE, but only four-repeat tau forms inclusions in PSP, GGT and CBD; PiD is a three-repeat tau proteinopathy.

$\mathrm{AD}$ is defined by the presence of abundant intraneuronal tau inclusions and extracellular $A \beta$ deposits [41]. Cognitive impairment and neurodegeneration correlate better with the number and distribution of tau inclusions than with $A \beta$ assemblies. Although silver staining, amyloid dyes and antibodies that label tau assemblies in post mortem brains have been available for many years, PET ligands that detect tau inclusions in living subjects have only recently been developed. Cross-sectional studies have shown that lower brain volumes are more strongly associated with tau-PET than A $\beta$-PET signals [8]. A prospective longitudinal study has 
indicated that the levels of tau-PET signal predict rates of brain atrophy [29], in agreement with neuropathological studies [4].

First-generation tau PET probes can be divided into three major chemical groups, with ${ }^{18} \mathrm{~F}$-THK5351 [22], ${ }^{18} \mathrm{~F}$-flortaucipir [7] and ${ }^{11} \mathrm{C}-\mathrm{PBB} 3$ (pyridinyl-butandienyl-benzothiazole 3) [35] being representative compounds. ${ }^{11} \mathrm{C}-\mathrm{PBB} 3$ is the most promising for visualizing inclusions made of only $3 \mathrm{R}$ or $4 \mathrm{R}$ tau isoforms. However, rapid conversion into a metabolite results in low entry of unmetabolized ${ }^{11} \mathrm{C}-\mathrm{PBB} 3$ into the brain. Recently, it has been shown that second-generation tau PET probe ${ }^{18}$ F-APN-1607, also called ${ }^{18}$ F-PMPBB3 (propanol modification of PBB3), a compound with high metabolic stability, labels brain regions with abundant tau inclusions in AD and PSP [26, 46]. Here we used cryoEM structure determination to identify the binding sites of APN-1607 in PHFs and SFs from AD.

\section{Materials and methods}

\section{Clinical history and neuropathology}

Tau filaments were extracted from the frontal cortex of neuropathologically confirmed cases of AD with sarkosyl (case 2 in reference [15]) or by affinity chromatography [28]. Occipital cortex from a previously described individual (case 18 in reference [15]) with PCA was used for tau filament extraction with sarkosyl. Tau filaments were extracted with sarkosyl from the entorhinal cortex or hippocampus of 3 previously undescribed individuals with PART. Case 1, with definite PART, was a male who died aged 88 following a 2 -year history of progressive memory loss. In addition to tau changes, prominent large- and smallvessel cerebrovascular disease associated with white matter loss was observed; small, healed cerebral infarcts were present in the frontal cortex and basal ganglia. There was substantial atrophy of the visual system, consistent with a history of long-term blindness. This individual, who suffered numerous head injuries, was declared legally blind at age 21. Signs of a healed subdural haemorrhage were seen at autopsy. $\alpha$-Synuclein and TDP-43 inclusions were not observed. Case 2, with definite PART, was a male who died aged 65 with cognitive impairment. There were signs of extensive small vessel disease; TDP-43 inclusions were present in nerve cells and glial cells, mostly in some subcortical regions. $\alpha$-Synuclein inclusions were not detected. Case 3, with possible PART, was a female who died aged 88 following a 2-year history of progressive memory loss. $\alpha$-Synuclein or TDP-43 inclusions were not observed. Additional findings are summarized in Table 1.

\section{Histology, immunohistochemistry and silver staining}

Histology and immunohistochemistry were carried out as described [45]. Briefly, $8 \mu \mathrm{m}$ paraffin-embedded tissue sections were treated for antigen retrieval, prior to overnight incubation with primary antibodies at room temperature. The sections were counterstained with haematoxylin/eosin and Luxol fast blue. Primary antibodies were: RD3 tau [10], anti-4R tau [12], AT8 [36], anti-phospho TDP-43 [23, 27], anti-A $\beta$ 6F/3D [33] and anti-A $\beta$ 4G8 [32] (Supplemental Fig. 1, online resource). Secondary antibodies were either ImmPRESS HRP anti-rabbit or anti-mouse IgG (Vector, Burlingame, CA), with 3,3'-diaminobenzidine used as
Table 1 Cases of Alzheimer's disease, posterior cortical atrophy and primary age-related tauopathy used for sarkosyl extraction and cryo-EM

\begin{tabular}{llllllll}
\hline Diagnosis & Gender & $\begin{array}{l}\text { Age at } \\
\text { death }(\mathrm{y})\end{array}$ & Brain region & Tau stage & $\mathrm{A} \beta$ phase & $\begin{array}{l}\text { A } \beta \text { angi- } \\
\text { opathy } \\
\text { scale }\end{array}$ & $\begin{array}{l}\text { APOE } \\
\text { haplo- } \\
\text { types }\end{array}$ \\
\hline AD & F & 82 & Frontal cortex & VI & 5 & 3 & $\varepsilon 4 / \varepsilon 4$ \\
PCA & M & 63 & Occipital cortex & VI & 5 & 3 & $\varepsilon 4 / \varepsilon 4$ \\
PART1 & M & 88 & Hippocampus & IV & 0 & 0 & $\varepsilon 2 / \varepsilon 3$ \\
PART2 & M & 65 & Entorhinal cortex & I & 0 & 0 & $\varepsilon 3 / \varepsilon 3$ \\
PART3 & F & 88 & Hippocampus & III & 2 & 3 & $\varepsilon 3 / \varepsilon 4$ \\
\hline
\end{tabular}

Alzheimer's disease (AD) (case 2) and posterior cortical atrophy (PCA) (case 18) have been described [15]. Primary age-related tauopathy (PART) cases 1-3 have not been described before. There were no known disease-causing mutations in MAPT. PART cases $1-3$ had a heterozygous T185S change in TMEM106B (gene encoding transmembrane protein 106B). PART case 1 had a heterozygous R62H change in TREM2 (gene encoding triggering receptor expressed on myeloid cells 2) and a heterozygous N357S change in TIA1 (gene encoding cytotoxic granule-associated RNA-binding protein). Whole-exome sequencing did not detect mutations known to cause $\mathrm{AD}$, Parkinson's disease, frontotemporal dementias or amyotrophic lateral sclerosis. PART case 1 was also heterozygous for mutations C195F and D1005V in CRB1 (gene encoding crumbs homologue 1). Biallelic mutations in CRB1 cause retinal disease [31], consistent with the development of blindness in this individual. His mother also suffered from retinal disease and was blind. Braak tau stages, Thal $\mathrm{A} \beta$ phases and Olichney $\mathrm{A} \beta$ angiopathy scales were determined according to references $[4,39,47]$ 
chromogen. Some sections were silver-impregnated using the method of Gallyas-Braak [5].

\section{Fluorescence staining of tau deposits with APN-1607}

Fluorescence staining of brain sections from cases of AD, PCA and PART with APN-1607 were done as described $[40,46]$. Briefly, deparaffinized $6 \mu \mathrm{m}$ sections were incubated in $50 \%$ ethanol containing $25 \mu \mathrm{M}$ APN-1607 at room temperature for $30 \mathrm{~min}$. They were rinsed in $50 \%$ ethanol for $5 \mathrm{~min}$., washed twice in water for $3 \mathrm{~min}$. and mounted in non-fluorescent media (Vectashield, Vector Laboratories). Fluorescence images were captured using a DM4000 microscope (Leica) equipped with a custom filter cube, as described [46].

\section{Whole-exome sequencing}

Target enrichment made use of the SureSelectTX human all-exon library (V6, 58 megabase pairs; Agilent) and highthroughput sequencing was carried out using a HiSeq 4000 (sx75 base-pair paired-end configuration; Illumina). Bioinformatics analyses were performed as described [17].

\section{Extraction of tau filaments}

Sarkosyl-insoluble material was extracted from hippocampus and cortex (frontal, occipital and entorhinal) [19]. Tissues were homogenized in $10 \mathrm{ml} / \mathrm{g}$ tissue of extraction buffer consisting of $10 \mathrm{mM}$ Tris- $\mathrm{HCl}$, pH 7.5, $10 \%$ sucrose, $0.8 \mathrm{M} \mathrm{NaCl}, 5 \mathrm{mM}$ EDTA, $1 \mathrm{mM}$ EGTA and a protease and phosphatase inhibitor (Thermo Fisher). Homogenates were spun at 20,000 $\mathrm{g}$ for $20 \mathrm{~min}$. and supernatants retained. Pellets were homogenized in $5 \mathrm{ml} / \mathrm{g}$ extraction buffer and centrifuged at 20,000 $\mathrm{g}$ for $20 \mathrm{~min}$. Both supernatants were combined, brought to $1 \%$ sarkosyl and incubated for $60 \mathrm{~min}$. at room temperature. Following a $60 \mathrm{~min}$. centrifugation at $100,000 \mathrm{~g}$, pellets were resuspended in $250 \mu \mathrm{l} / \mathrm{g}$ extraction buffer and spun at 20,000 $\mathrm{g}$ for $20 \mathrm{~min}$. The resulting supernatants were centrifuged at $100,000 \mathrm{~g}$ for $1 \mathrm{~h}$. For cryo$\mathrm{EM}$, pellets were resuspended in $25 \mu \mathrm{l} / \mathrm{g}$ tissue of $20 \mathrm{mM}$ Tris-HCl, pH 7.5, $100 \mathrm{mM} \mathrm{NaCl}$. Tau filaments were immunopurified from the frontal cortex of two individuals with neuropathologically confirmed AD (cases a and b), as described [28].

\section{Binding of APN-1607 to PHFs and SFs.}

APN-1607 was synthesised as described [26]. A $10 \mathrm{mM}$ solution was prepared using anhydrous dimethyl sulfoxide (DMSO, Thermo Fisher) and stored at $-20^{\circ} \mathrm{C}$. Tau filaments were sarkosyl-extracted from frontal cortex of an individual who had died with a neuropathologically confirmed diagnosis of AD (case 2 in reference [15]) and incubated with $100 \mu \mathrm{M}$ APN-1607 in $20 \mathrm{mM}$ Tris-HCl, pH 7.5, $100 \mathrm{mM} \mathrm{NaCl}, 1 \% \mathrm{DMSO}$, for $3 \mathrm{~h}$ at room temperature $(25 \mu \mathrm{l} / \mathrm{g}$ tissue). The concentration of APN-1607 was $2.5 \mathrm{nmol} / \mathrm{g}$ tissue. Controls were incubated with buffer containing 1\% DMSO. Given the photosensitivity of APN-1607, these experiments were carried out in the dark, except for a yellow light source with a wavelength greater than $525 \mathrm{~nm}$.

\section{Electron cryo-microscopy}

Following a $1 \mathrm{~min}$ spin at $3000 \mathrm{~g}, 3 \mu \mathrm{l}$ of supernatant were placed on glow-discharged holey carbon grids (Quantifoil Au R1.2/1.3, 300 mesh) and plunge-frozen in liquid ethane using an FEI Vitrobot Mark IV. The images of PART case 2 were acquired using a Falcon III detector in linear mode on a Glacios cryo-transmission electron microscope (Thermo Fisher) at $200 \mathrm{kV}$. All other images were acquired using a Gatan K2 summit detector in counting mode on a Titan Krios (Thermo Fisher) at $300 \mathrm{kV}$. A GIF quantum energy filter (Gatan) was used with a slit width of $20 \mathrm{eV}$ to remove inelastically scattered electrons. Further details are given in Supplemental Fig. 2, online resource.

\section{Helical reconstruction}

Movie frames were motion-corrected and dose-weighted using the motion correction implementation of RELION [51]. Aligned and non-dose weighted micrographs were used to estimate the contrast transfer function (CTF) by CTFFIND-4.1 [42]. Other image-processing steps were performed using helical reconstruction in RELION [24, 43]. Filaments were picked manually and extracted using an inter-box distance of $14.1 \AA$. For reference-free $2 \mathrm{D}$ classification, segments with a box size comprising an entire helical crossover were downscaled by a factor of 2-4 to speed up calculations. Different types of filament were separated by reference-free 2D classifications and segments contributing to suboptimal $2 \mathrm{D}$ averages discarded. For immunopurified AD tau filaments, as well as sarkosyl-extracted tau filaments from PART cases 1 and 3, initial 3D models were constructed de novo from 2D class averages comprising an entire helical crossover using the relion_helix_inimodel2d program [43]. For the other datasets, EMD-0259 (PHF) and EMD-0260 (SF) (from AD case 2 in reference [15]) were used as the initial 3D models. Helical twists were estimated either by crossover distances from 2D class averages, or according to those reported [15]. Segments for 3D autorefinement were then re-extracted using a box size comprising approximately $33 \%$ of the helical crossover, without downscaling. With an initial 3D model that was low-pass filtered to $10 \AA$, 3D auto-refinement was carried out for 
several rounds with optimization of helical twist and rise, after reconstructions had shown separation of $\beta$-strands along the helical axis. Since PHF protofilaments are related by an approximate $2_{1}$ screw symmetry [18], additional helical symmetry was applied from the initial 3D refinements. We then performed Bayesian polishing and CTF refinement, followed by 3D classification without further image alignment, to remove segments that yielded suboptimal 3D reconstructions. To further improve the structure of the $\beta$-helix region of SF protofilament 2 from PART case 3 , we used focused 3D classification (with signal subtraction) of V337-S356 without further image alignment. Final reconstructions were sharpened using standard post-processing procedures in RELION [51]. Overall resolution estimates were calculated from the Fourier shell correlations (FCSs) at 0.143 between two independently refined half-maps, using phase-randomisation to correct for convolution effects of a generous, soft-edged solvent mask that extended to $20 \%$ of the height of the box. Using the relion_helix_toolbox program [43], helical symmetries were imposed on the postprocessed maps, which were then used for model building and refinement. For further details, see Supplemental Figs. 2 and 3 , online resource.

\section{Model building and refinement}

6HRE for PHFs [15], 6HRF for SFs [15] and 6NWP for CTE type I filaments [16] were used as initial references. Models containing five $\beta$-sheet rungs were refined in real-space by PHENIX [1] using local symmetry to keep all rungs identical. For SFs from PART case 3, the models were refined against the map before focused 3D classification, followed by real-space refinement in COOT [13] of only the turns between $\beta$-strands 4 and 5 against the map after focused 3D classification. MolProbity [6] was used for model validation. To confirm the absence of overfitting, FSC curves between one half-map and the model, which was refined against the other half-map, were checked. Additional details are given in Supplemental Figs. 2 and 3, online resource.

\section{Quantitation of extra densities}

For each dataset, 3 independent refinements were carried out by dividing the cryo-EM images 3 times into different half-sets in RELION. They used the same references and parameters, without optimisation of helical symmetry. Each reconstruction was post-processed, with a low-pass filter of $3.0 \AA$ and a B-factor of $-70 \AA^{2}$ for the PHF maps, and a low-pass filter of $3.55 \AA$ and a B-factor of $-55 \AA$ for the SF maps. Helical symmetry was applied to post-processed half-maps using the relion_helix_toolbox program [43]. The reconstructions with a box size of 256 pixels and a pixel size of $1.15 \AA$ were up-sampled to 558 pixels, from which the central rung could be extracted in an integer number of pixels (i.e., 9 pixels) along the helical axis. The central 18 slices were used for analysis. Binary masks were then created for the different binding sites; a solvent region extending 29 pixels around the entire filaments; and for residues 349-370 (binding sites 1, 2a, 2b, 3, 4) or 318-340 (binding sites 5, $6 \mathrm{a}, 6 \mathrm{~b})$ of tau. Relative densities were calculated as follows:

Relative density (binding site $)=[\operatorname{Max}($ binding site $)-\operatorname{Avg}($ solvent $)]$ :

$$
[\operatorname{Max}(\operatorname{protein})-\operatorname{Avg}(\text { solvent })] \text {, }
$$

where: $\operatorname{Max}$ (binding site) is the highest pixel value within the mask for the binding site; Avg(solvent) is the average pixel value in the solvent region; $\operatorname{Max}$ (protein) is the highest pixel value in the protein density for residues 349-370 and 318-340 of tau (using the numbering of the 441 amino acid isoform of human brain tau).

\section{Results and discussion}

\section{Structures of tau filaments from Alzheimer's disease with APN-1607}

We added APN-1607 to sarkosyl-insoluble tau filaments from the frontal cortex of case 2 of AD (in reference [15]), prior to imaging by cryo-EM (+APN-1607). We used the PET ligand at $2.5 \mathrm{nmol} / \mathrm{g}$. The $B_{\max }$ of ${ }^{18} \mathrm{~F}-\mathrm{PM}$-PBB3 for tau aggregates in $\mathrm{AD}$ frontal cortex has been reported to be $5.7 \mathrm{nmol} / \mathrm{g}$ [46]. The mean concentration of sarkosylinsoluble tau is around $1 \mathrm{nmol} / \mathrm{g}$ cerebral cortex from AD [49]. The approximate molar APN-1607: tau ratio was therefore 2.5. As controls, we imaged tau filaments following the addition of only buffer (-APN-1607). By using helical reconstruction in RELION $[24,43]$, we determined the structures of PHFs to $3.0 \AA$ (+APN-1607) and $2.8 \AA$ (-APN-1607). There were fewer SFs, giving resolutions of $3.6 \AA$ (+APN1607) and $3.2 \AA$ (-APN-1607). These maps enabled us to study additional densities that were well separated from those of tau (Fig. 1; Supplemental Fig. 3, online resource).

For PHFs, we observed additional densities in the + APN1607 map compared to the -APN-1607 map (Fig. 1a, c, e), with the overall structure being unchanged (Fig. 2a). The root mean-square deviation between refined atomic coordinates in both maps was $0.4 \AA$. In the -APN-1607 map, densities in these grooves were weaker, approaching the intensity levels of noise features in the solvent region, suggesting that the extra densities in the +APN-1607 map corresponded to the PET ligand. In a difference map, these sites showed up as well-resolved peaks when displayed at a threshold of 11 standard deviations (Fig. 1c).

One additional density was present in the groove between the side chains of R349 and Q351 from $\beta 6$ of the Alzheimer 
Fig. 1 Cryo-EM maps of PHFs and SFs from Alzheimer's disease with APN-1607. a, b Cryo-EM maps of tau filaments without (-APN1607) and with (+APN-1607) PET ligand. c, d, Overlay of positive (orange) and negative (purple) difference maps and -APN1607 maps (grey). SF protofilaments 1 and 2 are labelled as PF-1 and PF-2. Amino acids R349, Q351 and K353 are indicated, with the C-shaped cavities outlined by stippled lines. The thresholds in the difference maps were 11 standard deviations for PHFs and 8 standard deviations for SFs. e, Alzheimer tau protofilament core with APN-1607 binding sites. Major binding sites 1, 2a, $2 \mathrm{~b}$ and 3 are shown in orange; minor binding sites $4,5,6 \mathrm{a}$ and $6 \mathrm{~b}$ are indicated in yellow

tau fold (binding site 1). Two additional densities were observed in the groove between the side chains of Q351 and K353 (binding sites $2 \mathrm{a}$ and $2 \mathrm{~b}$ ), possibly reflecting the width of this groove (Figs. 1, 2b). It is possible that side-toside interactions between APN-1607 molecules at sites 2a and $2 \mathrm{~b}$, e.g. $\pi$-stacking between the $\pi$-electron-conjugated backbone of APN-1607, are required for binding at site $2 b$, and that APN-1607 may not be able to bind to site $2 \mathrm{~b}$ when site 2a is empty. Despite a similar hydrophobic environment, the density at site 1 was weaker than at site $2 \mathrm{a}$. These same grooves between the side chains that formed binding sites $1,2 \mathrm{a}$ and $2 \mathrm{~b}$ were previously identified by molecular docking among the predicted binding sites for multiple tau PET ligands [21, 38].

In SFs, protofilament 1 was better defined than protofilament 2. Nevertheless, we observed similar extra densities in both protofilaments at binding sites $1,2 \mathrm{a}$ and $2 \mathrm{~b}$, as well as an additional elongated density within the C-shaped cavity (binding site 3) (Fig. 1b, d, e). When comparing the + APN1607 and -APN-1607 maps, we observed a $7^{\circ}$ difference in the orientation of $\beta 7$ for protofilament 1 and a $3^{\circ}$ difference for protofilament 2 (Fig. 2c, d). This suggests that the binding of APN-1607 induces a conformational change that enlarges the size of the C-shaped cavity in SFs. Even in the absence of APN-1607, this cavity is wider in SFs than in PHFs. Thus, an approximate $12^{\circ}$ difference in the turn between $\beta 6$ and $\beta 7$ was observed when comparing PHFs and SFs in the -APN-1607 maps (Fig. 2e). When displaying the difference maps for SFs at a threshold of 8 standard deviations, all the binding sites showed up as well-resolved peaks and the conformational changes resulting from APN-1607 binding led to additional differences in SFs (Fig. 1d).

To rule out that the weak densities at sites 1,2a and $2 \mathrm{~b}$ were caused by random noise, we performed 3 map refinements for each dataset, by dividing the cryo-EM images 3 times into different half-sets. We then quantified the densities at binding sites $1,2 \mathrm{a}$ and $2 \mathrm{~b}$ in each of the 6 half-set reconstructions relative to the densities of tau (see Methods). The additional densities at the various binding sites were weaker than the densities of tau. For PHFs, the additional densities were approximately $40 \%$ relative to the density of tau for site $1,60 \%$ for site $2 \mathrm{a}$ and $40 \%$ for
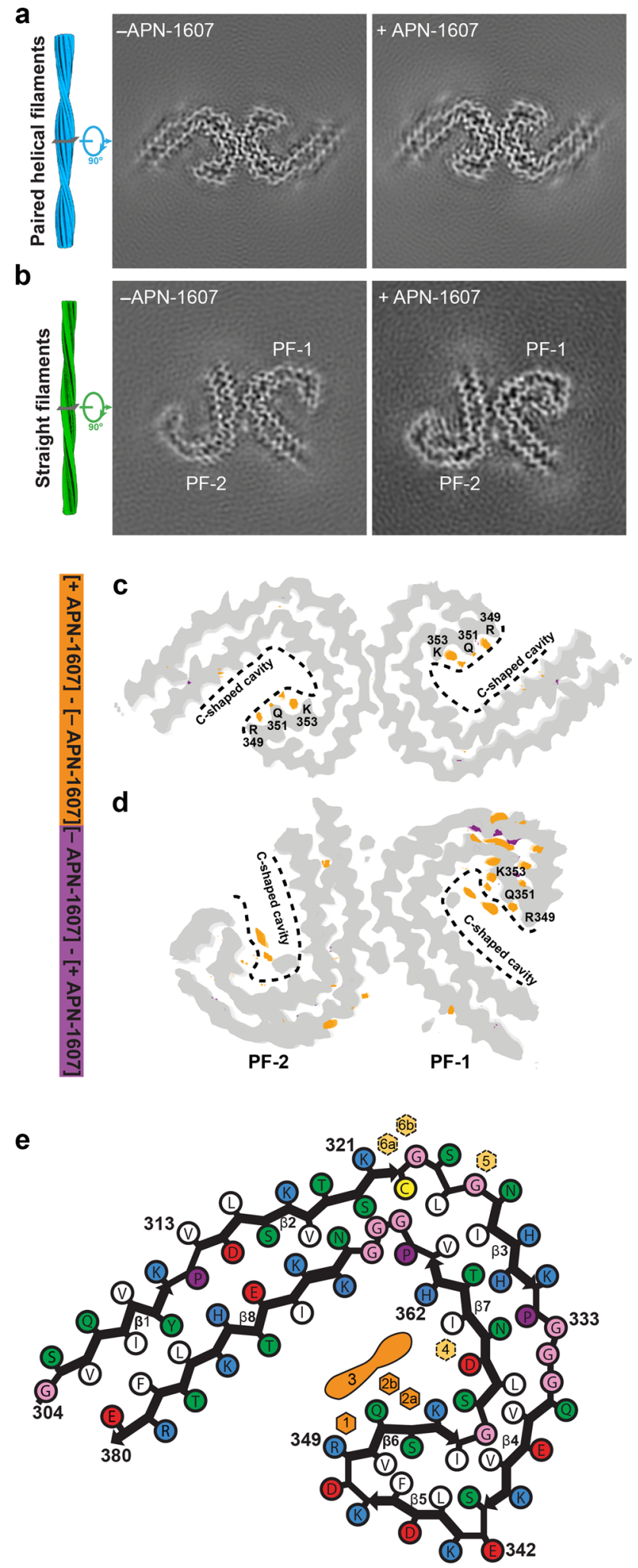

site $2 \mathrm{~b}$. For SFs, the relative densities were approximately $55 \%, 50 \%$ and $45 \%$, respectively. In the absence of APN1607, they were 5-15\% in PHFs and SFs (Fig. 3a). 


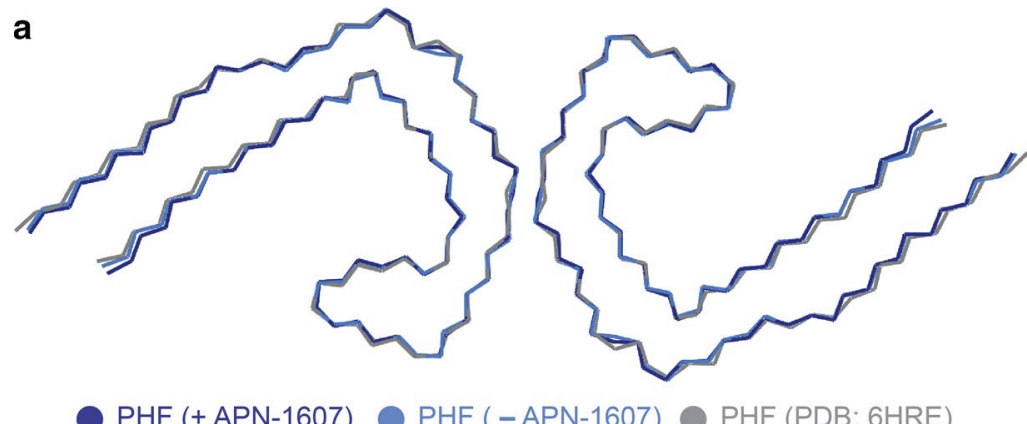

$\mathrm{PHF}(+\mathrm{APN}-1607) \bigcirc \mathrm{PHF}(-\mathrm{APN}-1607) \bigcirc \mathrm{PHF}(\mathrm{PDB}: 6 \mathrm{HRE})$

C
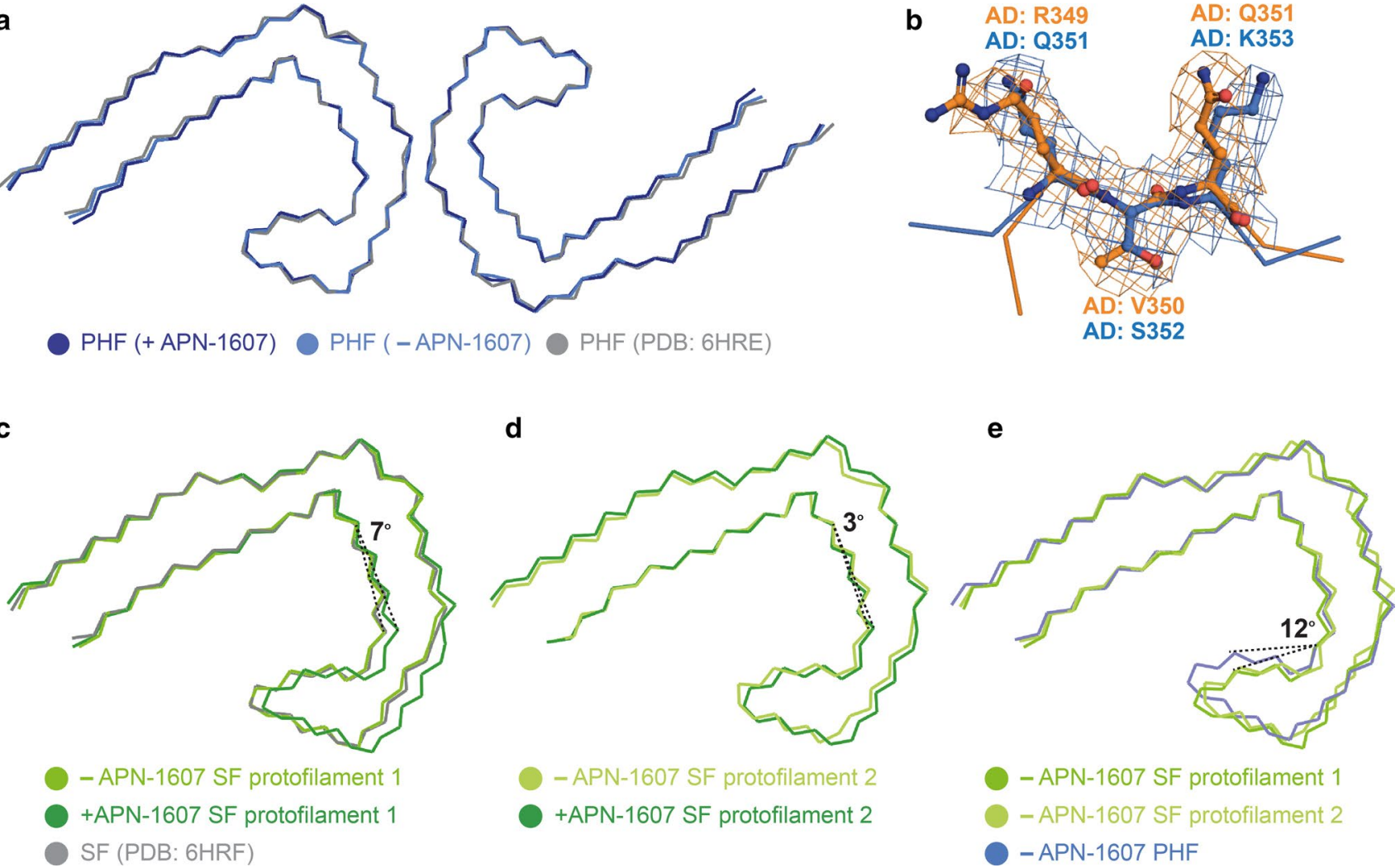

Fig. 2 Alzheimer tau folds with and without APN-1607. a, Overlay of the structures of PHFs (+APN-1607; -APN-1607; PDB: 6HRE). b Overlay of R349-Q351 (binding site 1) and Q351-K353 (binding site 2) of PHFs. Flanking residues are shown as main-chain traces. c, d, Overlay of the structures of SF protofilaments 1 (c) and 2 (d) (+APN-

To exclude that sarkosyl binds to the same sites and interferes with APN-1607 binding, we determined the cryo-EM structures of tau filaments immunopurified from the frontal cortex of two neuropathologically confirmed cases of AD (cases a and b) (Supplemental Fig.4, online resource). For case (a), cryo-EM micrographs showed PHFs and SFs in a ratio of approximately $4: 1$. Using helical reconstruction in RELION [24, 43], we determined the structures of PHFs to $2.9 \AA$ and SFs to $7.0 \AA$ A resolution (Supplemental Figs. 3b and $4 \mathrm{a}$, online resource). For case (b), we observed mainly PHFs and determined their structure to $3.2 \AA$ resolution (Supplemental Figs. $3 b$ and $4 b$, online resource). We found no significant differences between sarkosyl-extracted and immunopurified filaments (Supplemental Fig. 4c, online resource). Immunopurified filaments also showed weak densities in both grooves. Therefore, we exclude sarkosyl as the reason for weak additional densities in the -APN-1607 structures. It is possible that molecules from the brain bind with low occupancy at these sites.

APN-1607 (Fig. 3b) fits into the elongated densities observed at binding sites $1,2 \mathrm{a}$ and $2 \mathrm{~b}$, suggesting that it binds parallel to the long helical axis of amyloid filaments
1607; -APN-1607; PDB: 6HRF). e, Overlay of SF protofilaments 1 and 2, and the PHF protofilament (-APN-1607). The structures were aligned based on residues 363-380 of tau (in the numbering of the 441 amino acid isoform of human brain tau)

(Fig. 3c, d). A similar mode of binding has been reported for thioflavin-T, Congo red and luminescent conjugated oligothiophenes [25, 34, 44]. It remains to be seen if other tau PET ligands bind in the same grooves of the Alzheimer tau fold. Binding sites 1 and 2 have Q351 in common, which adopts an extended conformation. Although the orientation of its amide headgroup cannot be determined at the current resolution, it probably forms a hydrogen-bonded ladder along the filament axis, providing a rigid partition between sites 1 and 2 . The positively charged headgroups of R349 and K353 are kept at a distance from Q351 by electrostatic interactions with the carboxylic groups of D348 and D358, creating predominantly hydrophobic grooves with polar edges as the binding sites. It follows that bound APN1607 with a hydrophobicity $\log p$ value of 4.01 is stabilized mostly by hydrophobic interactions; occasional hydrogen bonds may form between its polar atoms and the side chains and/or carbonyl oxygens of tau. Additional densities are mostly featureless along the filament axis, suggesting that they do not obey helical symmetry. Therefore, the relative positions of individual APN-1607 molecules in the direction parallel to the helical axis cannot be determined from the 
Fig. 3 Binding of APN-1607 to PHFs and SFs. a, Relative densities at binding sites $1,2 \mathrm{a}$, $2 \mathrm{~b}$ and 3 in cryo-EM maps of PHFs and SFs. Means, standard deviations and individual values of 6 half-set reconstructions are shown. Unpaired twotailed t-test: $* * * * p<0.0001$. b, Chemical structure of APN1607 (PM-PBB3). c, d, Top views and side views of the extra densities in the APN-1607 binding sites of PHF (c) and SF (d) maps. The models of APN1607 are shown near these extra densities at the same scale

a Paired helical filaments
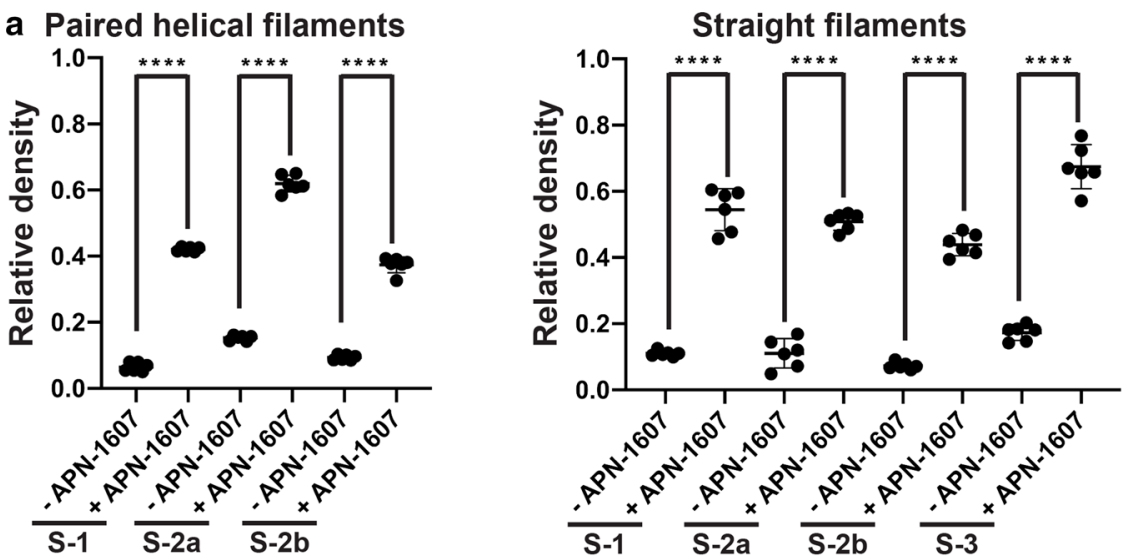

b<smiles>CNc1ccc(/C=C/C=C/c2nc3ccc(OCC(O)CF)cc3s2)cn1</smiles>

APN-1607 (PM-PBB3)

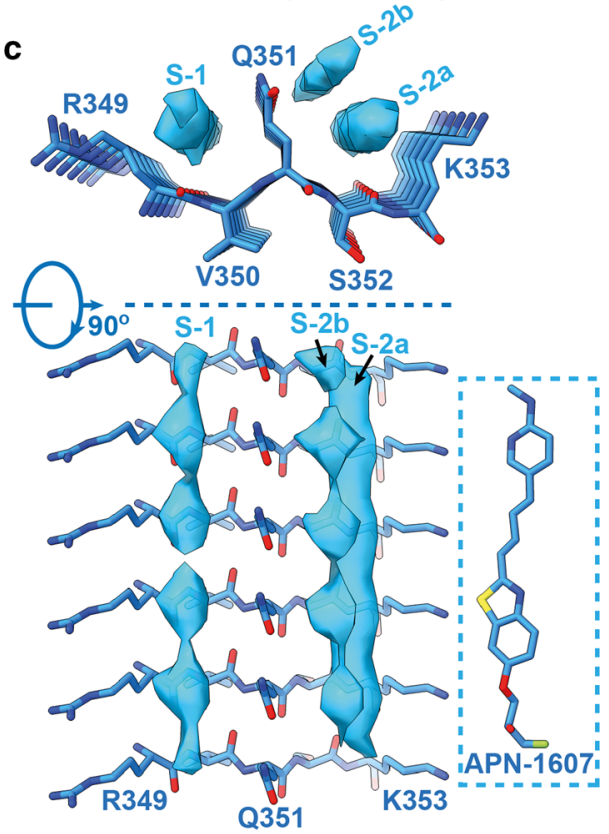

d
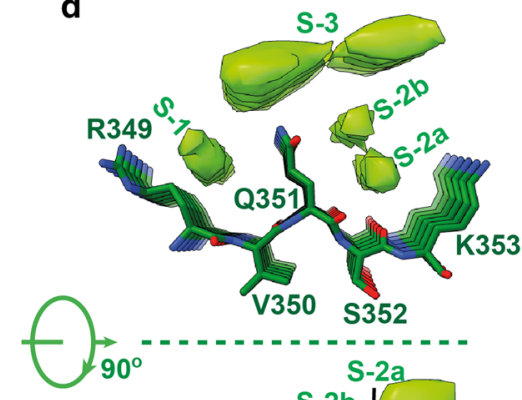

S-2a

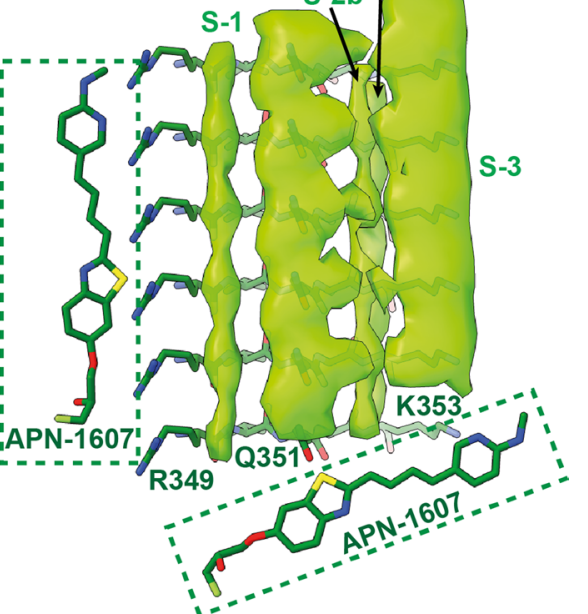

cryo-EM maps. In addition, the blurring that results from the helical averaging in the reconstruction process complicates the interpretation of the relative densities in terms of the stoichiometry of ligand binding at the different sites.

The elongated shape of the additional density at binding site 3, with a density level of approximately $70 \%$ relative to that of tau (Fig. 3a, d), suggests that in SFs APN-1607 is able to bind in a direction nearly perpendicular to the helical axis, although the exact orientation and relative positions of individual molecules cannot be determined from the cryoEM map. This site was not identified by molecular docking $[21,38]$. At binding site 3 , APN-1607 is probably stabilized on one side by hydrophobic interactions with APN-1607 molecules at binding sites 1 and $2 \mathrm{~b}$. On the other side, APN1607 faces two additional densities of unknown nature. They have also been observed in the cryo-EM maps of PHFs and SFs from the frontal cortex of 4 cases of AD [15]. The finding that in PHFs there is less space between these additional densities and the $\beta$-helix at the tip of the $\mathrm{C}$ may explain why binding site 3 is not occupied. Weaker densities were also found in grooves other than those of binding sites 1 and 2 . We refer to these minor binding sites as 4, 5, 6a and $6 \mathrm{~b}$ (Fig. 1e; Supplemental Fig. 5 , online resource). In SFs, binding at sites $5,6 \mathrm{a}$ and $6 \mathrm{~b}$ was only seen in protofilament 2. Binding at site $6 \mathrm{~b}$ was not observed in PHFs. A statistical analysis of the relative densities of all APN-1607 binding 
sites in PHFs and SFs from AD is given in Supplemental Fig. 6, online resource. The presence of an additional major APN-1607 binding site in SFs suggests that the ratio between PHFs and SFs could influence ligand binding, in particular the $B_{\max }$.

The tau protofilament folds of CTE, PiD and CBD differ from that of $\mathrm{AD}[14,16,18,50]$. Binding studies have suggested that APN-1607 may also be able to image tau inclusions of PiD and CBD [46]. It will be important to identify the binding sites of APN-1607 in tau filaments from these diseases. In the Pick fold, molecular docking has identified a potential binding site for APN-1607 in the groove between R349 and Q351 of tau [37, 46].

\section{Structures of tau filaments from posterior cortical atrophy and primary age-related tauopathy}

PCA, a variant of $A D$, is characterized by an initial decline in visual processing, with the early presence of abundant tau inclusions and $\mathrm{A} \beta$ assemblies in the occipital cortex [3]. PART [9] has been described as a pathological continuum, ranging from localized entorhinal and hippocampal tau inclusions in individuals with normal or mildly impaired cognition to widespread tau inclusions in patients with tangle-only dementia (TD) [2, 9, 48]. It has also been proposed that PART is part of the events that lead to the presence of abundant plaques and tangles in neocortex, with a clinical picture of $\mathrm{AD}$ [11].

We determined cryo-EM structures of tau filaments from the occipital cortex of PCA (Fig. 4a). PHFs and SFs were present in a ratio of 9:1. Reconstructions calculated in RELION [24, 43] were identical to those determined previously from the frontal cortex of $\mathrm{AD}[15,18]$. The resolutions were $3.6 \AA$ for PHFs and $5.2 \AA$ for SFs (Fig. 4a; Supplemental Fig. 3b, online resource). We also imaged two cases of definite PART and one case of possible PART by cryo-EM and immunohistochemistry (Fig. 4b; Supplemental Figs. 7-10, online resource). Silver-positive tangles were present in the hippocampus of cases 1 and 3 and the entorhinal cortex of case 2. Sarkosyl-insoluble tau filaments from the hippocampus of PART case 1, who suffered from long-term blindness, had 26\% PHFs and 61\% SFs; $13 \%$ were a
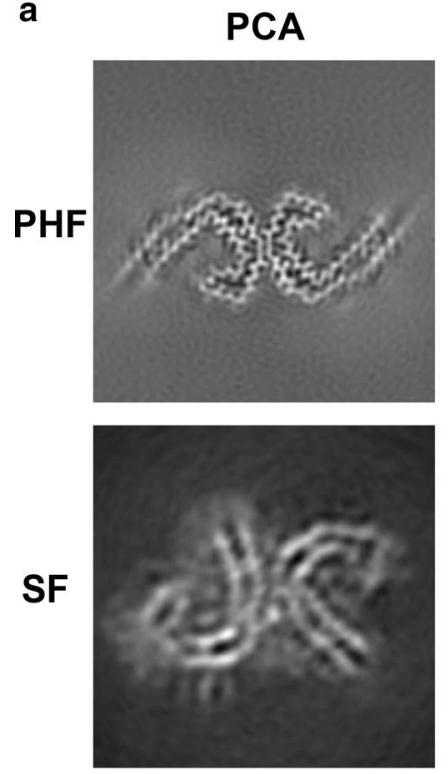

b
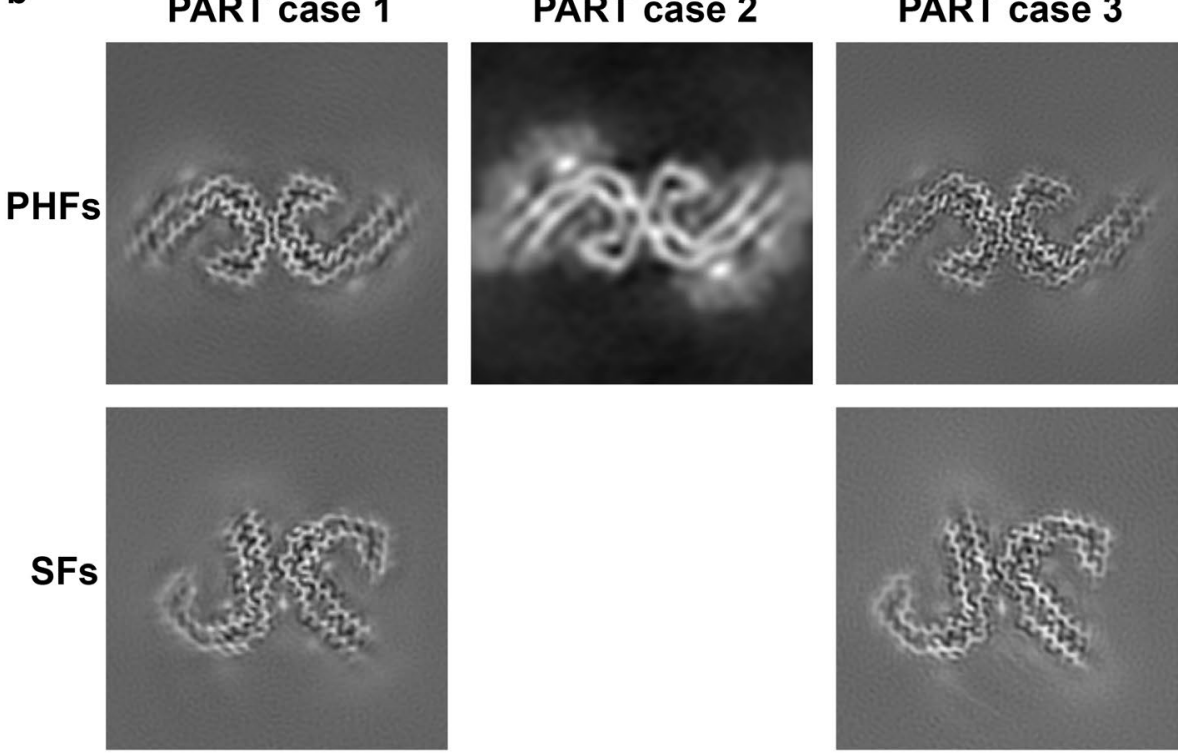

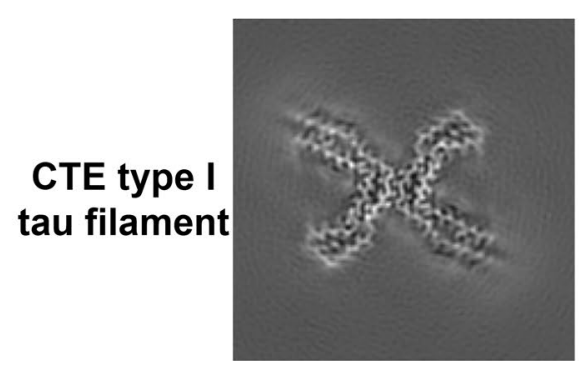

Fig. 4 Cryo-EM maps of tau filaments from posterior cortical atrophy and primary age-related tauopathy. a PHF and SF from the occipital cortex of a case of PCA. b PHFs and SFs from the hippocampus of
PART cases 1 and 3 and PHF from the entorhinal cortex of PART case $2 ; 13 \%$ of filaments from PART case 1 were CTE type I tau filaments 


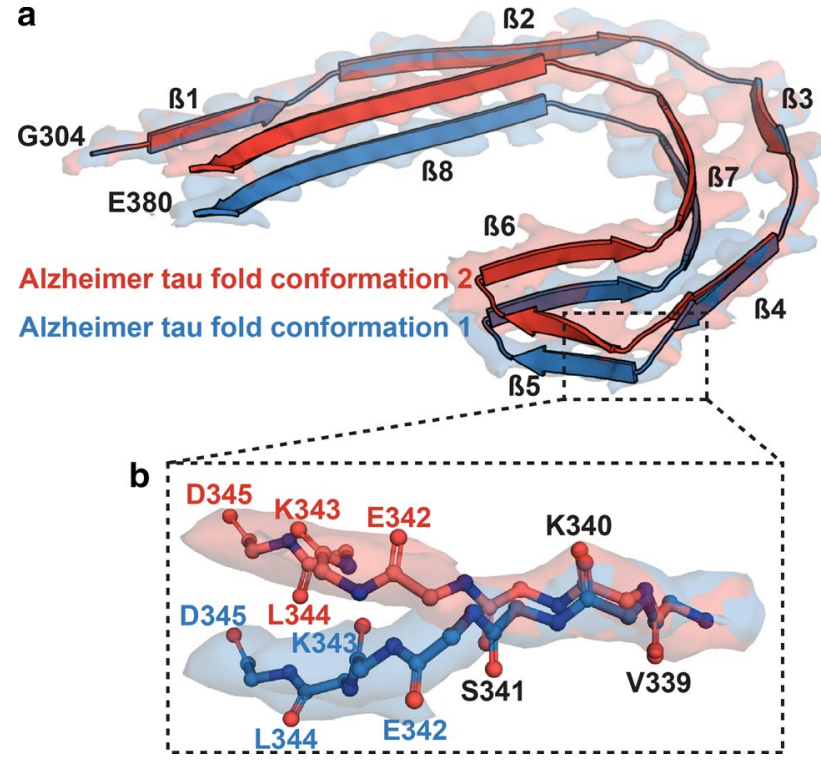

Fig. 5 Two conformations of Alzheimer tau fold in SF protofilament 2 from PART case 3. a Overlay of two conformations of the Alzheimer tau fold from SF protofilament 2. The structures were aligned based on amino acids 304-340 of tau (in the numbering of the 441 amino acid isoform of human brain tau). b Close-up view showing that the difference between conformations was a flip of the peptide groups between E342 and its flanking residues, S341 and K343

CTE type I filaments [16]. The presence of CTE type I filaments was consistent with a history of head trauma and the presence of tau inclusions around blood vessels in occipital lobe (Supplemental Fig. 7, online resource). PART case 2 had only PHFs, whereas PART case 3 had $42 \%$ PHFs and $58 \%$ SFs. TDP-43 inclusions were present in subcortical brain regions of PART case 2 (Supplemental Fig. 8, online resource). $A \beta$ deposits were observed in cortical regions of PART case 3, but not PART case 1 or 2 (Supplemental Fig. 10, online resource). We determined the structures of PHFs to resolutions of: $3.2 \AA$ (PART case 1), $5.8 \AA$ (PART case 2) and $2.8 \AA$ (PART case 3 ). For SFs, the resolutions were: $2.8 \AA$ (PART case 1) and $2.7 \AA$ (PART case 3 ).

In previous maps $[15,18]$, the density of SF protofilament 2 was not of sufficient quality to allow unambiguous chain tracing in the direction along the helical axis, in particular the $\beta$-helix region. Given the overall appearance of the density in the plane perpendicular to the helical axis, where the interactions between amino acid side chains shaped the Alzheimer tau fold, we assumed that the main chain would adopt the same fold in both protofilaments. At $2.7 \AA$, the cryo-EM map from PART case 3 has the highest known resolution for a SF (Figs. 4b and 5). We carried out further 3D classification that showed two distinct conformations (Fig. 5a). They were distinguished by flips of the peptide bonds between E342 and its flanking residues, S341 and K343 in conformation 2, which led the turn between strands 4 and 5 of the $\beta$-helix to connect to strands in adjacent rungs, with the rest of the structure being unchanged (Fig. 5b). Conformation 1 was identical to the tau fold of the PHF and $\mathrm{SF}$ protofilament 1. Conformation 2 was more similar to the CTE tau fold. The mixing of conformations suggests that in a substantial proportion of SFs, protofilament 2 can give rise to a subtype of the Alzheimer tau fold that is distinct from the fold of the PHF and from that of the SF protofilament 1. This could play a role in the asymmetric packing of SF protofilaments.
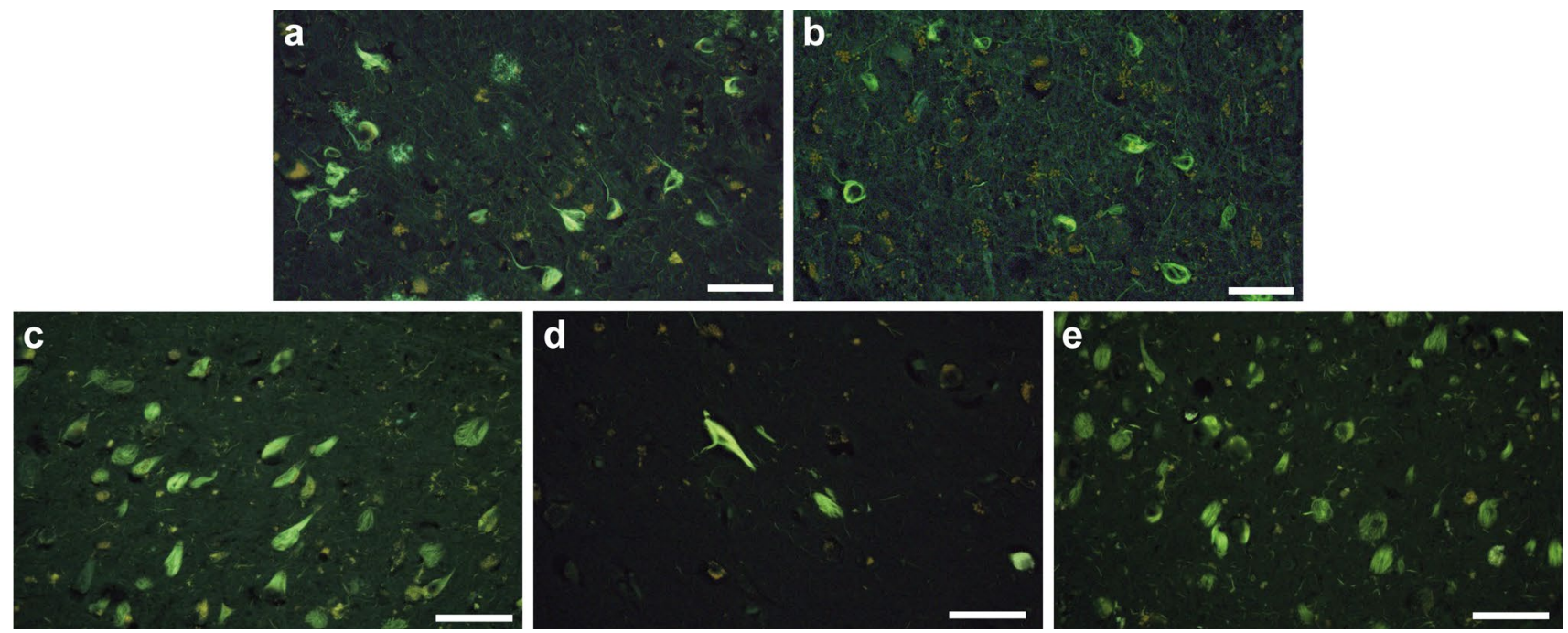

Fig. 6 Staining of tau inclusions with fluorescent APN-1607. Sections from the frontal cortex of $\mathrm{AD}$, occipital cortex of PCA, hippocampus of PART cases 1 and 3, and entorhinal cortex of PART case 2 were stained with APN-1607. The same cases of the disease were used as for cryo-EM. a AD; b PCA; c PART case 1; d PART case 2; e PART case 3. Scale bar, $50 \mu \mathrm{m}$ 
These findings establish that the Alzheimer tau fold can form in the absence of $\mathrm{A} \beta$ deposits and support the observation that homogenates from the brains of patients with $\mathrm{AD}$ and PART had similar seeding activities [30].

\section{Staining of tau inclusions from Alzheimer's disease, posterior cortical atrophy and primary age-related tauopathy with APN-1607}

Since APN-1607 is fluorescent, its reactivity with tau inclusions can be investigated in brain sections [46]. APN-1607 labelled numerous nerve cell body inclusions in tissue sections from the cases of AD (Fig. 6a), PCA (Fig. 6b) and PART (Fig. 6c-e) used for cryo-EM, providing a link, not only with AD, but also with PCA and PART. Some neuritic plaques were also labelled (Fig. 6a). Triple staining of brain sections with APN-1607, an antibody against phosphorylated tau and Gallyas-Braak silver staining has shown binding of APN-1607 to tau inclusions in AD [46]. In PART, the number of stained inclusions was proportional to the Braak stages. Thus, PART case 2, with tau pathology at Braak stage I, had the smallest number of fluorescent inclusions (Fig. 6d). It follows that APN-1607 may also be useful as a PET ligand for the tau inclusions of PCA and PART.

\section{Conclusion}

Taken together, our findings show that APN-1607 binds in the $\beta$-helix region of PHFs and SFs. This work establishes the use of cryo-EM to study the binding of small molecule compounds to amyloid filaments, which will allow the design of new PET ligands with increased specificity and binding activity for a range of neurodegenerative diseases.

Supplementary Information The online version contains supplementary material available at https://doi.org/10.1007/s00401-021-02294-3.

Acknowledgements We thank the families of the patients for donating brain tissues; T. Nakane for help with RELION; T. Darling and J. Grimmett for help with high-performance computing; F. Epperson, M.H. Jacobsen, U. Kuederli and R.M. Richardson for support with neuropathology. M.G. is an Honorary Professor in the Department of Clinical Neurosciences of the University of Cambridge and an Associate Member of the UK Dementia Research Institute. This work is dedicated to the memory of Peter Davies, Ph.D. (Litwin Zucker Center for Research on Alzheimer's disease, Feinstein Institute for Medical Research, Manhasset, NY), who provided immunopurified filaments from AD brains. It was supported by the UK Medical Research Council (MC_ UP_A025_1013, to S.H.W.S. and MC_U105184291, to M.G.), the European Union (EU/EFPIA/Innovative Medicines Initiative [2] Joint Undertaking IMPRIND, project 116060, to M.G.), the US National Institutes of Health (P30-AG010133, to B.G., and U01-NS110437, to B.G. and R.V.), the Department of Pathology and Laboratory Medicine, Indiana University School of Medicine and the Japan Agency for Medical Research and Development (JP20dm0207072, to M.H.). We acknowledge Diamond light Source for access and support of the cryo-EM facilities at the UK's national electron Bio-Imaging Centre
(eBIC), under proposal EM17434-41, funded by the Wellcome Trust, the MRC and the BBSRC. This study was supported by the MRC-LMB electron microscopy facility.

Author contributions K.L.N., R.V., H.J.G. and B.G. identified patients, performed neuropathology and carried out genomic characterization; Y.S. extracted tau filaments from the brains of individuals with $\mathrm{AD}$ and PART; B.F. extracted tau filaments from the brain of an individual with PCA; P.T. and M.-K.J. provided APN-1607; N.S. and M.H. stained tissue sections with APN-1607; Y.S. performed cryo-EM of AD tau filaments without and with APN-1607, of immunopurified tau filaments from $\mathrm{AD}$ (case 2) and of tau filaments from PART cases 1-3. Y.S. and S.H.W.S. analyzed these cryo-EM data; B.F. performed cryo-EM of immunopurified tau filaments from AD (case 1) and of tau filaments from the case with PCA; A.E., J.M., Y.S., B.F. and S.H.W.S. analyzed these cryo-EM data; Y.S., A.G.M. and S.H.W.S. analyzed the additional densities in + APN-1607 and -APN-1607 maps; Y.S. built the atomic models; S.H.W.S. and M.G. supervised the project; all authors contributed to the writing of the manuscript.

\section{Declarations}

Conflict of interest M.H. holds a patent related to APN-1607. P.T., M.H. and M.-K.Y. are equity holders in APRINOIA. The other authors declare no competing financial interests.

Open Access Cryo-EM maps have been deposited in the Electron Microscopy Data Bank (EMDB) under accession numbers EMD12551 for +APN-1607 PHF, EMD-12552 for +APN-1607 SF, EMD12553 for PHF of PCA case, EMD-12549 and EMD-12550 for PHF and SF of PART case 3, respectively. Refined atomic models have been deposited in the Protein Data Bank (PDB) under accession numbers 7NRV for + APN-1607 PHF, 7NRX for + APN-1607 SF, 7NRQ, 7NRS and 7NRT for PHF, SF conformation 1 and SF conformation 2 of PART case 3, respectively. Whole-exome sequencing data have been deposited in the National Institute on Aging Alzheimer's Disease Data Storage Site (NIAGADS; https://www.niagads.org), under accession number NG00101.

Open Access This article is licensed under a Creative Commons Attribution 4.0 International License, which permits use, sharing, adaptation, distribution and reproduction in any medium or format, as long as you give appropriate credit to the original author(s) and the source, provide a link to the Creative Commons licence, and indicate if changes were made. The images or other third party material in this article are included in the article's Creative Commons licence, unless indicated otherwise in a credit line to the material. If material is not included in the article's Creative Commons licence and your intended use is not permitted by statutory regulation or exceeds the permitted use, you will need to obtain permission directly from the copyright holder. To view a copy of this licence, visit http://creativecommons.org/licenses/by/4.0/.

\section{References}

1. Adams PD, Safonine PV, Bunkóczi G, Chen VB, Davis IW, Echols $\mathrm{N}$ et al (2010) PHENIX: a comprehensive Python-based system for macromolecular structure solution. Acta Cryst D 66:213-221

2. Bancher C, Jellinger KA (1994) Neurofibrillary tangle predominant form of senile dementia of Alzheimer type: a rare subtype in very old subjects. Acta Neuropathol 88:565-570 
3. Benson DF, Davis RJ, Snyder BD (1988) Posterior cortical atrophy. Arch Neurol 45:789-793

4. Braak H, Braak E (1991) Neuropathological stageing of Alzheimer-related changes. Acta Neuropathol 82:239-259

5. Braak E, Braak H (1991) Demonstration of amyloid deposits and neurofibrillary changes in whole brain sections. Brain Pathol $1: 213-216$

6. Chen VB, Arendall WB 3rd, Headd JJ, Keedy DA, Immormino RM, Kapral GJ et al (2010) MolProbity: all-atom structure validation for macromolecular crystallography. Acta Cryst D 66:12-21

7. Chien DT, Bahri S, Szardenings AK, Walsh JC, Mu F, Su MY et al (2013) Early clinical PET imaging results with the novel PHF-tau radioligand [F-18]-T807. J Alzheimer's Dis 34:457-468

8. Cho H, Choi JY, Hwang MS, Lee JH, Kim YJ, Lee HM et al (2016) Tau PET in Alzheimer disease and mild cognitive impairment. Neurology 87:375-383

9. Crary JF, Trojanowski JQ, Schneider JA, Abisambra JF, Abner EL, Alafuzoff I et al (2014) Primary age-related tauopathy (PART): a common pathology associated with human aging. Acta Neuropathol 128:755-766

10. de Silva R, Lashley T, Gibb G, Hanger D, Hope A, Reid A et al (2003) Pathological inclusion bodies in tauopathies contain distinct complements of tau with three or four microtubule-binding repeat domains as demonstrated by new specific monoclonal antibodies. Neuropathol Appl Neurobiol 29:288-302

11. Duyckaerts C, Braak H, Brion JP, Buée L, Del Tredici K, Goedert $\mathrm{M}$ et al (2015) PART is part of Alzheimer disease. Acta Neuropathol 129:749-756

12. Ebashi M, Toru S, Nakamura A, Kamei S, Yokota T, Hirokawa $\mathrm{K}$ et al (2019) Detection of AD-specific four repeat tau with deamidated asparagine residue 279-specific fraction purified from $4 \mathrm{R}$ tau polyclonal antibody. Acta Neuropathol 138:163-166

13. Emsley P, Lohkamp B, Scott WG, Cowtan K (2010) Features and development of coot. Acta Cryst D 66:486-501

14. Falcon B, Zhang W, Murzin AG, Murshudov G, Garringer HJ, Vidal RA et al (2018) Structures of filaments from Pick's disease reveal a novel tau protein fold. Nature 561:137-140

15. Falcon B, Zhang W, Schweighauser M, Murzin AG, Vidal R, Garringer HJ et al (2018) Tau filaments from multiple cases of sporadic and inherited Alzheimer's disease adopt a common fold. Acta Neuropathol 126:699-708

16. Falcon B, Zivanov J, Zhang W, Murzin AG, Garringer HJ, Vidal RA et al (2019) Novel tau filament fold in chronic traumatic encephalopathy encloses hydrophobic molecules. Nature 568:420-423

17. Farlow JL, Robak LA, Hetrick K, Bowling K, Boerwinkle E, Coban-Akademir ZH et al (2016) Whole-exome sequencing in familial Parkinson disease. JAMA Neurol 73:68-75

18. Fitzpatrick AWP, Falcon B, He S, Murzin AG, Murshudov G, Garringer HJ et al (2017) Cryo-EM structures of tau filaments from Alzheimer's disease. Nature 547:185-190

19. Goedert M, Spillantini MG, Cairns NJ, Crowther RA (1992) Tau proteins of Alzheimer paired helical filaments: abnormal phosphorylation of all six brain isoforms. Neuron 8:159-168

20. Goedert M, Eisenberg DS, Crowther RA (2017) Propagation of tau aggregates and neurodegeneration. Annu Rev Neurosci 40:189-210

21. Goedert M, Yamaguchi Y, Mishra SK, Higuchi M, Sahara N (2018) Tau filaments and the development of positron emission tomography tracers. Front Neurol 9:70

22. Harada R, Okamura N, Furumoto S, Furukawa K, Ishiki A, Tomita $\mathrm{N}$ et al (2016) ${ }^{18} \mathrm{~F}$-THK5351: a novel PET radiotracer for imaging neurofibrillary pathology in Alzheimer disease. J Nucl Med 57:208-214
23. Hasegawa M, Arai T, Nonaka T, Kametani F, Yoshida M, Hashizume Y et al (2008) Phosphorylated TDP-43 in frontotemporal lobar degeneration and amyotrophic lateral sclerosis. Ann Neurol 64:60-70

24. He S, Scheres SHW (2017) Helical reconstruction in RELION. J Struct Biol 198:163-176

25. Herrmann US, Schütz AK, Shirani H, Huang D, Saban D, Nuvolone $\mathrm{M}$ et al (2015) Structure-based drug design identifies polythiophenes as antiprion compounds. Sci Transl Med 7:299ra123

26. Hsu JL, Lin KJ, Hsiao IT, Huang KL, Liu CH, Wu HC et al (2020) The imaging features and clinical associations of a novel tau PET tracer- ${ }^{18}$ F-APN1607 in Alzheimer disease. Clin Nucl Med 45:747-756

27. Inukai $\mathrm{Y}$, Nonaka $\mathrm{T}$, Arai $\mathrm{T}$, Yoshida $\mathrm{M}$, Hashizume $\mathrm{Y}$, Beach TG et al (2008) Abnormal phosphorylation of Ser409/410 of TDP-43 in FTLD-U and ALS. FEBS Lett 582:2899-2904

28. Jicha GA, O’Donnell A, Weaver C, Angeletti R, Davies P (1999) Hierarchical phosphorylation of recombinant tau by the pairedhelical filament-associated protein kinase is dependent on cyclic AMP-dependent protein kinase. J Neurochem 72:214-224

29. Joie RL, Visani AV, Baker SL, Brown JA, Bourakova V, Cha J et al (2020) Prospective longitudinal atrophy in Alzheimer's disease correlates with the intensity and topography of baseline tauPET. Sci Transl Med 12:eaau5732

30. Kaufman SK, Del Tredici K, Thomas TL, Braak H, Diamond MI (2018) Tau seeding activity begins in the transentorhinal/entorhinal regions and anticipates phospho-tau pathology in Alzheimer's disease and PART. Acta Neuropathol 136:57-67

31. Khan KN, Robson A, Mahroo OAR, Arno G, Inglehearn CF, Armengol $\mathrm{M}$ et al (2018) A clinical and molecular characterisation of CRB1-associated maculopathy. Eur J Hum Genet 26:687-694

32. Kim KS, Miller DL, Sapienza VJ, Chen CMJ, Bai C, GrundkeIqbal I et al (1988) Production and characterization of monoclonal antibodies reactive to synthetic cerebrovascular amyloid peptide. Neurosci Res Commun 2:121-130

33. Kim KS, Wen GY, Bancher C, Chen CMJ, Hong H, Wisniewski HM et al (1990) Detection and quantitation of amyloid $\beta$-peptide with 2 monoclonal antibodies. Neurosci Res Commun 7:113-122

34. Krebs MRH, Bromley EHC, Donald AM (2005) The binding of thioflavin-T to amyloid fibrils: localisation and implications. J Struct Biol 149:30-37

35. Maruyama M, Shimada H, Suhara T, Shinotoh H, Ji B, Maeda J et al (2013) Imaging of tau pathology in a tauopathy mouse model and in Alzheimer patients compared to normal controls. Neuron 79:1094-1108

36. Mercken M, Vandermeeren M, Lübke U, Six J, Boons J, Van de Voorde A et al (1992) Monoclonal antibodies with selective specificity for Alzheimer tau are directed against phosphatase-sensitive epitopes. Acta Neuropathol 84:265-272

37. Mishra SK, Yamaguchi Y, Higuchi M, Sahara N (2021) Pick's tau fibril shows multiple distinct PET probe binding sites: Insights from computational modelling. Int J Mol Sci 22:349

38. Murugan NA, Nordberg A, Agren H (2018) Different positron emission tomography tau tracers bind to multiple binding sites on the tau fibril: insight from computational modeling. ACS Chem Neurosci 9:1757-1767

39. Olichney JM, Hansen LA, Hofstetter CR, Grundman M, Katzman R, Thal LJ (1995) Cerebral infarction in Alzheimer's disease is associated with severe amyloid angiopathy and hypertension. Arch Neurol 52:702-708

40. Ono M, Sahara N, Kumata K, Ji B, Ni R, Koga S et al (2017) Distinct binding of PET ligands PBB3 and AV-1451 to tau fibril strains in neurodegenerative tauopathies. Brain 140:764-780

41. Peng C, Trojanowski JQ, Lee VMY (2020) Protein transmission in neurodegenerative disease. Nat Rev Neurol 16:199-212 
42. Rohou A, Grigorieff N (2015) CTFFIND4: fast and accurate defocus estimation from electron micrographs. J Struct Biol 192:216-221

43. Scheres SHW (2020) Amyloid structure determination in RELION-3.1. Acta Crystallogr D 76:94-101

44. Schütz AK, Soragni A, Hornemann S, Aguzzi A, Ernst M, Böckmann A et al (2011) The amyloid-Congo red interface at atomic resolution. Angew Chem Int Ed Engl 50:5956-5960

45. Spina S, Farlow MR, Unverzagt FW, Kareken DA, Murrell JR, Fraser $\mathrm{G}$ et al (2008) The tauopathy associated with mutation +3 in intron 10 of Tau: characterization of the MSTD family. Brain 131:72-89

46. Tagai K, Ono M, Kubota M, Kitamura S, Takahata K, Seki C et al (2021) High-contrast in vivo imaging of tau pathologies in Alzheimer's and non-Alzheimer's disease tauopathies. Neuron 109:42-58

47. Thal DR, Rüb U, Orantes M, Braak H (2002) Phases of $\mathrm{A} \beta$-deposition in the human brain and its relevance for the development of Alzheimer's disease. Neurology 58:1791-1800
48. Ulrich J, Spillantini MG, Goedert M, Dukas L, Staehelin H (1992) Abundant neurofibrillary tangles without senile plaques in a subset of patients with senile dementia. Neurodegeneration $1: 257-284$

49. Wesseling H, Mair W, Kumar M, Schlaffner CN, Tang S, Beerepoot $\mathrm{P}$ et al (2020) Tau PTM profiles identify patient heterogeneity and stages of Alzheimer's disease. Cell 183:1699-1713

50. Zhang W, Tarutani A, Newell KL, Murzin AG, Matsubara T, Falcon B et al (2020) Novel tau filament fold in corticobasal degeneration. Nature 580:283-287

51. Zivanov J, Nakane T, Forsberg BO, Kimanius D, Hagen WJ, Lindahl E et al (2018) New tools for automated high-resolution cryoEM structure determination in RELION-3. eLife 7:e42166

Publisher's Note Springer Nature remains neutral with regard to jurisdictional claims in published maps and institutional affiliations. 